\title{
Qualidade de vida e desesperança em familiares de dependentes químicos
}

\author{
Antonio Teulberto Mesquita Aragão ${ }^{1}$ - Universidade Federal de São Paulo, Brasil \\ Elizabete Milagres - Universidade Federal de São Paulo, Brasil \\ Neliana Buzi Figlie - Universidade Federal de São Paulo, Brasil
}

\begin{abstract}
Resumo
Medir o nível de Qualidade de Vida e Desesperança em um grupo de mulheres residentes na periferia de São Paulo atendidas em um centro de intervenção e apoio que convivem com um dependente químico em seus lares. Participaram desta amostra 56 mulheres. A entrevista foi realizada individualmente no início do tratamento no serviço, sendo investigados os transtornos psiquiátricos na amostra, segundo critérios diagnósticos do CID-10. Os instrumentos de avaliação aplicados foram: entrevista para obtenção de dados demográficos; o levantamento do CCEB; WHOQOL-bref que mede Qualidade de Vida e BHS (Beck Hopelessness Scale) que mede nível de desesperança, acrescido de avaliação psiquiátrica. Foi constatado que $39 \%$ das mulheres apresentam desesperança mínima; 44\% leve; 16\% moderada/grave. Na Qualidade de Vida destacou-se o domínio Físico $(x=14,45)$, seguido pelo domínio Social $(x=13,26)$, Psicológico $(x=12,57)$ e Meio Ambiente $(x=10,68)$. No tocante aos transtornos psiquiátricos, $23 \%(n=13)$ apresentaram depressão e $5 \%(n=3)$ apresentaram transtorno de ansiedade. A maioria dos familiares possui nível de desesperança satisfatório, porém o grupo com desesperança moderada/grave desperta preocupações e demonstra a necessidade de intervenção, principalmente quando os dados são analisados com a presença de transtornos psiquiátricos.

Palavras-chave: relações familiares; transtornos relacionados ao uso de substâncias; qualidade de vida.
\end{abstract}

\begin{abstract}
To measure the quality of life and hopelessness of a group of women living in the outskirts of Sao Paulo attended at a center for assistance and support living with a drugs addicts in their homes. Participated in this sample, 56 women. They were individually interviewed in beginning of the treatment to investigate psychiatric disorders in the sample, according to diagnoses criteria of CID-10. The scales used on this study were interviews in order to gather demographical data; the CCEB survey; the WHOQOL-bref that measures life quality, the BHS (Beck Hopelessness Scale), that measures the level of hopelessness and a psychiatric assessment. It was noted that $39 \%$ of women presented minimal hopelessness, $44 \%$ light and 16\% moderate / severe hopelessness. In Life Quality the physical domain was outstanding $(x=14,45)$, followed by the social domain $(x=13,26)$, the psychological domain $(x=12,57)$ and the environmental domain $(x=10,68)$. Concerning the psychiatric diagnosis, $23 \%(n=13)$ showed depression and $5 \%(n=3)$ showed anxiety. The majority of relatives presented satisfactory level of hopelessness. Nonetheless, the group with moderate/severe hopelessness demands attention and shows intervention need, especially when data is analyzed considerating the psychiatric diagnosis.

Keywords: family relations; substance-related disorders; quality of life.
\end{abstract}

Quality of life and hopelessness of relatives of addicted

\section{Introdução}

É sabido que a dependência de substâncias psicoativas sofre influência de fatores psicológicos, sociológicos, culturais e espirituais e os mesmos desempenham um importante papel na causa, curso e resultados do transtorno (Figlie, Fontes, Moraes \& Payá, 2004; Schenker \& Minayo, 2004). Destarte é possível afirmar que a dependência química repercute não só no usuário de substâncias, mas também nos familiares que convivem com ele.

Estudos demonstram que o sucesso do tratamento relacionado ao uso de substâncias está relacionado com a adequada intervenção dos familiares (Carr, 2004). Alguns autores descrevem a família como um sistema em que cada membro

\footnotetext{
${ }^{1}$ Endereço para correspondência:

Avenida Antonio Carlos, 6627, Campus da Pampulha, Depto. de Psicologia, Universidade Federal de Minhas Gerais, 31270-901

Email: teo_aragao@yahoo.com.br
}

está interligado de forma que a mudança em uma das partes provoca reverberação em toda a estrutura e cada um, por sua vez, é agente participante desse fenômeno (Henggeler, Melton \& Smith, 1992; Schenker \& Minayo, 2004), indicando que o indivíduo deve ser compreendido não só no contexto da sua individualidade, mas também familiar.

A convivência dos familiares com o usuário, como uma via de mão dupla, também é afetada na medida em que a dependência química evolui e se desenvolve. Estudos evidenciam que esposas de maridos dependentes de álcool apresentam sofrimento e um apelo para uma vida de resignação e sacrifícios acompanhada por sentimentos de solidão, frustrações e tristezas em virtude da deficiência desses maridos no exercício do papel de pai e esposo (Tobo \& Zago, 2005). Tempier e colaboradores (2006) constataram que as esposas de alcoolistas apresentam sinais de ansiedade, depressão, agressividade e prejuízos 
cognitivos como indicativos de alto estresse psicológico.

Kearns-Bodkin e Leonard (2005) verificaram que existe uma associação entre o uso do álcool por parte de um dos cônjuges e a qualidade da relação conjugal, com enfraquecimento do vínculo conjugal mediante o decorrer do tempo de exposição à doença. O alcoolismo do parceiro foi um dos fatores mais frequentes em episódios de agressão contra mulheres, sendo que $72 \%$ da amostra estudada apresentou depressão, 78\% sintomas de ansiedade e insônia e 39\% pensaram em suicídio (Adeodato \& cols., 2005).

A desesperança dos familiares, objeto de investigação neste estudo, é considerada como o "nexo causal entre depressão e suicídio" (Cunha, 2001; Minkoff, Bergman, Beck \& Beck, 1973) e pode funcionar como um desencadeante de alguns comportamentos e transtornos psíquicos. Segundo Stotland (1969), as pessoas desesperançadas acreditam: 1) que nada sairá bem para elas, 2) que nunca terão sucesso no que tentarem fazer, 3) que suas metas importantes jamais poderão ser alcançadas e 4) que seus piores problemas nunca serão solucionados (Beck, 2001).

A qualidade de vida, segundo a Organização Mundial da Saúde (OMS), é a percepção do indivíduo de sua posição na vida, no contexto da cultura e sistema de valores nos quais ele vive e em relação aos seus objetivos, expectativas, padrões e preocupações (The WHOQOL Group, 1998).

O presente estudo tem o objetivo de avaliar o índice de desesperança e da qualidade de vida de um grupo de mulheres residentes na periferia de São Paulo assistidas em um centro de apoio e intervenção, que não são dependentes químicas, mas convivem em uma família que possui ao menos um dependente.

\section{Método}

\section{Local}

O CUIDA (Centro Utilitário de Intervenção e Apoio aos Filhos de Dependentes Químicos) está instalado no Jardim Ângela, periferia de São Paulo, região com alto índice de desemprego, violência, consumo de substâncias e tráfico de drogas (Fund. Seade; Secr. Est. Saúde, 2001-2005; Hinkly \& Laranjeira, 2002).

O CUIDA oferece assistência psiquiátrica e psicológica com o objetivo de estimular condutas saudáveis no inter-relacionamento familiar, envolvendo tanto a criança quanto os seus familiares, que recebem orientação e/ou psicoterapia familiar.

\section{Amostra}

Foi utilizada uma amostragem de conveniência dos casos que ingressaram no serviço no período de 2003 a 2005. Participaram dessa amostra 56 mulheres, esposas ou companheiras de dependentes químicos, sendo $77 \% \quad(n=43)$ de esposas/companheiras e $23 \%$ $(n=13)$ de outros familiares (mães $n=7$ e irmãs $\mathrm{n}=6$ ). Foram excluídos dessa análise 4 casos, 3 por se tratar do sexo masculino que poderiam atuar como variável de confusão, e 1 por estar com o questionário incompleto. Vale ressaltar que não ocorreram recusas na participação do estudo e que as mulheres estudadas não possuem diagnóstico de dependência química, com exceção do tabaco.

A entrevista foi realizada individualmente na primeira consulta no serviço, sendo verificado posteriormente, junto ao psiquiatra do serviço, as mulheres que apresentaram transtorno psiquiátrico segundo critérios diagnósticos do CID-10.

A maior parte da amostra tem entre 26 a 35 anos $(46 \%, n=26), 27 \%(n=15)$ tem idade entre 36 a 45 anos, 20\% (n=11) acima de 46 anos e $7 \%$ $(n=4)$ entre 15 a 25 anos. Com relação à escolaridade, $36 \%(n=20)$ têm ensino fundamental incompleto; $12 \% \quad(\mathrm{n}=7)$ têm fundamental completo, $29 \% \quad(\mathrm{n}=16)$ possuem ensino médio completo ou incompleto e $23 \%(n=13)$ da amostra são analfabetas. Em termos de renda familiar a maior parte da amostra recebe 1 salário mínimo $(48 \%, \mathrm{n}=27)$ e/ou entre 2 e 3 salários $(48 \%$, $\mathrm{n}=27$ ), sendo que a minoria recebe acima de 3 salários mínimos $(4 \%, n=2)$, caracterizando $22 \%$ $(n=12)$ das entrevistadas pertencentes a classe $C$; $73 \%(n=41)$ pertence a classe $D$; e $5 \%(n=3)$ a classe E. Por fim, 43\% ( $n=24)$ das entrevistadas possuem até 2 filhos; $30 \% \quad(n=17)$ possuem 3 filhos e $27 \%(n=15)$ possuem 4 ou mais filhos Tabela 1.

\section{Instrumentos}

A aplicação dos instrumentos foi realizada por uma assistente social e uma psicóloga, ambas treinadas para tal finalidade, após a avaliação clínica do serviço. As mesmas preenchiam o protocolo, que era aplicado individualmente, em virtude da dificuldade de escolarização da amostra em estudo. 
Tabela 1. Distribuição dos dados sociodemográficos de familiares de dependentes químicos $(\mathrm{N}=56)$

\begin{tabular}{|c|c|c|}
\hline Dados sócio-demográficos & $\%$ & $(n=56)$ \\
\hline \multicolumn{3}{|l|}{ 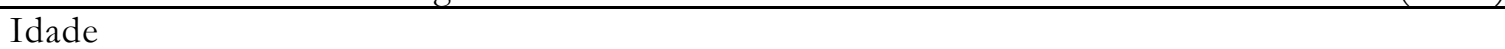 } \\
\hline 15 a 25 anos & 7 & 4 \\
\hline 26 a 35 anos & 46 & 26 \\
\hline 36 a 45 anos & 27 & 15 \\
\hline acima de 46 anos & 20 & 11 \\
\hline \multicolumn{3}{|l|}{ Raça } \\
\hline Branca & 63 & 35 \\
\hline Negra & 12 & 7 \\
\hline Parda & 25 & 14 \\
\hline \multicolumn{3}{|l|}{ Atual Situação de Trabalho } \\
\hline Do lar & 48 & 27 \\
\hline Desempregada & 16 & 9 \\
\hline Doméstica & 14 & 8 \\
\hline Assalariada & 13 & 7 \\
\hline Aposentada & 4 & 2 \\
\hline Outros & 5 & 3 \\
\hline \multicolumn{3}{|l|}{ Classe Social } \\
\hline C & 22 & 12 \\
\hline $\mathrm{D}$ & 73 & 41 \\
\hline $\mathrm{E}$ & 5 & 3 \\
\hline \multicolumn{3}{|l|}{ Grau de Parentesco } \\
\hline Esposas/companheiras & 77 & 43 \\
\hline Outros familiares & 23 & 13 \\
\hline \multicolumn{3}{|l|}{ Número de filhos } \\
\hline até 2 filhos & 43 & 24 \\
\hline 3 filhos & 30 & 17 \\
\hline 4 ou mais filhos & 27 & 15 \\
\hline
\end{tabular}

Foi utilizada uma entrevista estruturada para obtenção de dados demográficos (sexo, idade, renda familiar, etc.); levantamento dos Critérios de Classificação Econômica do Brasil (CCEB) (Ass. Nac. Emp. Pesq. ANEP, 1997), instrumento que estima o poder de compra dos indivíduos e famílias urbanas, com base no Levantamento Sócioeconômico (LSE) do IBOPE, classificandoos por classes econômicas A, B, C, D, e E; a Beck Hopelessness Scale (BHS) (Beck, 2001) é uma escala dicotômica, composta por 20 itens envolvendo cognições sobre desesperança que permitem avaliar a extensão das expectativas negativas a respeito do futuro imediato e remoto e a correção das respostas do questionário classifica os resultados como nível: mínimo, leve, moderado e grave; e o WHOQOL-Bref (Fleck, Louzada, Xavier, Chachamovich, Vieira, Santos \& Pinzon, 2000) é um instrumento desenvolvido pela Organização Mundial de Saúde (OMS) com 26 questões sobre qualidade de vida dividida em quatro domínios: físico, psicológico, relações sociais e meioambiente.
Ética

O estudo foi aprovado pelo Comitê de Ética para Pesquisas Médicas da Universidade Federal de São Paulo, Brasil. Todos os sujeitos receberam informações sobre a pesquisa, tendo assinado $\mathrm{O}$ formulário do consentimento informado antes de participar e sido assegurados do anonimato e da confidencialidade.

\section{Análise estatística}

Inicialmente as variáveis numéricas foram analisadas descritivamente através das medidasresumo (média, mediana, mínimo, máximo e desvio padrão) e as categóricas na forma de frequências absolutas e relativas.

As médias de WHOQOL-Bref em cada domínio por BHS foram comparadas por análise de Variância (ANOVA) seguida por comparações múltiplas. Os pressupostos da análise de variância são a normalidade e homocedasticidade (variâncias iguais entre os grupos). A normalidade foi verificada por meio dos testes de KolmogorovSmirnov nos resíduos do modelo da ANOVA e a 
homocedasticidade, pelo teste de Levene (Levene, 1960). Para os testes estatísticos foi utilizado um nível de significância de 5\%.

\section{Resultados}

Das mulheres estudadas constatou-se que $39 \% \quad(n=22)$ apresentam desesperança mínima; $45 \% \quad(n=25) \quad$ desesperança leve; $16 \% \quad(n=9)$ desesperança moderada/grave.

No nível de qualidade de vida é percebido por esta população como mais preservado o domínio físico $(x=14,45 ; \mathrm{DP}=2,82)$, seguido pelo domínio social $(x=13,26 ; D P=3,66)$, psicológico $(x=12,57 ; D P=2,94)$ e por fim o domínio meio ambiente $(\mathrm{x}=10,68$; $\mathrm{DP}=2,39)$.

Comparando os dados de qualidade de vida com desesperança constatamos uma associação entre os grupos com desesperança mínima com maior otimismo quanto a sua qualidade de vida e os grupos de desesperança moderada/grave com menor otimismo quanto a qualidade de vida, prevalecendo o domínio físico sobre os demais.

A média do WHOQOL-Bref no domínio físico para os que têm mínima desesperança $(\mathrm{x}=15,77 ; \mathrm{DP}=2,92)$ é superior à dos demais (leve: $x=13,81 ; D P=2,59$ e moderada/grave: $x=13,02$; $\mathrm{DP}=1,93)$, que foram consideradas similares. No aspecto psicológico verificou-se, para aqueles que têm desesperança mínima $(\mathrm{x}=14,52 ; \mathrm{DP}=2,76)$, um escore superior aos demais (leve: $\mathrm{x}=11,49$; $\mathrm{DP}=1,99$ e moderada/grave: $\mathrm{x}=10,81 \mathrm{DP}=3,14)$ que foram considerados similares - Tabela 2.

$\mathrm{Da}$ amostra total, $23 \% \quad(\mathrm{n}=13)$ apresentaram diagnóstico de depressão e $5 \%(n=3)$ diagnóstico de ansiedade, sendo que 16\% (n=9) dessas entrevistadas apresentaram desesperança moderada e grave.

Tabela 2. Comparações das médias da qualidade de vida por níveis de desesperança

\begin{tabular}{|c|c|c|c|c|c|c|c|}
\hline $\begin{array}{l}\text { Domínios da QDV e } \\
\text { categorização do BHS }\end{array}$ & Média & $\begin{array}{l}\text { Desvio } \\
\text { padrão }\end{array}$ & Mediana & $\begin{array}{l}\text { Erro } \\
\text { padrão }\end{array}$ & Mínimo & Máximo & $\mathrm{N}$ \\
\hline Físico & 14,45 & 2,82 & 14,29 & 0,38 & 7,43 & 20,00 & 56 \\
\hline Mínimo 0-4 & $15,77(1)$ & 2,92 & 16,00 & 0,62 & 9,71 & 20,00 & 22 \\
\hline Leve $5-8$ & $13,81^{(2)}$ & 2,59 & 14,29 & 0,52 & 7,43 & 18,29 & 25 \\
\hline Moderado/Grave 9 e mais & $13,02^{(2)}$ & 1,93 & 12,57 & 0,64 & 10,29 & 16,00 & 9 \\
\hline Psicológico & 12,57 & 2,94 & 12,33 & 0,39 & 5,33 & 20,00 & 56 \\
\hline Mínimo 0-4 & $14,52^{(1)}$ & 2,76 & 14,00 & 0,59 & 9,33 & 20,00 & 22 \\
\hline Leve $5-8$ & $11,49(2)$ & 1,99 & 11,33 & 0,40 & 8,00 & 16,00 & 25 \\
\hline Moderado/Grave 9 e mais & $10,81^{(2)}$ & 3,14 & 10,67 & 1,05 & 5,33 & 16,67 & 9 \\
\hline Social & 13,26 & 3,66 & 14,00 & 0,49 & 5,33 & 20,00 & 56 \\
\hline Mínimo 0-4 & 14,48 & 3,30 & 14,67 & 0,70 & 8,00 & 20,00 & 22 \\
\hline Leve $5-8$ & 12,59 & 3,95 & 13,33 & 0,79 & 5,33 & 18,67 & 25 \\
\hline Moderado/Grave 9 e mais & 12,15 & 3,16 & 12,00 & 1,05 & 6,67 & 16,00 & 9 \\
\hline Meio ambiente & 10,68 & 2,39 & 10,25 & 0,32 & 6,00 & 16,50 & 56 \\
\hline Mínimo 0-4 & 11,36 & 2,52 & 10,50 & 0,54 & 8,00 & 16,00 & 22 \\
\hline Leve $5-8$ & 10,22 & 2,45 & 10,00 & 0,49 & 6,00 & 16,50 & 25 \\
\hline Moderado/Grave 9 e mais & 10,28 & 1,58 & 10,00 & 0,53 & 8,00 & 13,50 & 9 \\
\hline Overall & 13,54 & 3,10 & 14,00 & 0,41 & 8,00 & 20,00 & 56 \\
\hline Mínimo & $15,09^{(1)}$ & 3,01 & 14,00 & 0,64 & 10,00 & 20,00 & 22 \\
\hline Leve & $12,64^{(2)}$ & 2,56 & 14,00 & 0,51 & 8,00 & 16,00 & 25 \\
\hline Moderado/Grave & $12,22^{(2)}$ & 3,38 & 12,00 & 1,13 & 8,00 & 18,00 & 9 \\
\hline
\end{tabular}

Observação: (1) e (2) representam grupos com médias distintas

\section{Discussão}

Castro e cols. (2007) em estudo realizado, afirmam que tabagistas graves têm prejuízos na qualidade de vida, no entanto ressalta que este mesmo grupo apresenta escores mais elevados de sintomas depressivos e de ansiedade. Adeodato e colaboradores (2005), em um trabalho realizado com mulheres vítimas de seus parceiros, concluíram que as mulheres vitimadas apresentaram escores compatíveis com uma má qualidade de vida e sintomas depressivos demonstrados no inventário de Beck (Gorestein \& Andrade, 1998). O autor observa que as agressões 
contra as mulheres ocorriam na sua maioria dentro de casa, quando o parceiro estava sob o efeito do álcool, o que confirma os argumentos levantados neste trabalho, de que a presença de um dependente químico dentro de casa está associada com distúrbios na harmonia e na convivência familiar afetando os familiares do mesmo.

Lima (2002), em seu estudo de qualidade de vida em pacientes do sexo masculino dependentes de álcool, conclui que quanto maior o grau de consumo com a bebida maior o prejuízo em qualidade de vida; no entanto observa que diversos autores que estudaram $\mathrm{o}$ assunto não alcançaram resultados consistentes, mesmo quando os resultados foram comparados com uma população de não-dependentes. Os autores observaram que muitos usuários sentem-se satisfeitos com sua qualidade de vida apesar da não-concordância das pessoas à sua volta, o que sugere que o alcoolista, nesse caso, associa qualidade de vida apenas como a percepção de saúde de um modo geral e a dor corporal.

No nosso trabalho os resultados alcançados foram considerados satisfatórios quanto à percepção de qualidade de vida e desesperança. Esse fato corrobora o conceito de que, embora a avaliação de qualidade de vida seja influenciada pelos acontecimentos externos, ela é uma experiência fundamentalmente subjetiva, motivada pelo estado mental, pela personalidade e pelas expectativas de cada um (Lima, 2002; Orley \& cols., 1998) ou seja, as mulheres objeto deste estudo definem a sua própria qualidade de vida adequada apesar de outros estudos afirmarem o contrário. Edwards e cols. (1999) concluíram que com o decorrer do tempo a esposa do dependente químico percebe que a situação piora e não muda. Passa então a desenvolver um sentimento de desesperança, ocorrendo a diminuição ou interrupção do contato sexual, distanciamento e sentimentos de medo, raiva, culpa, além de vivenciar problemas financeiros e domésticos.

Os resultados da BHS mostram que $83 \%$ $(n=47)$ das mulheres pesquisadas possuem desesperança mínima e leve, o que nos leva a considerar que essas famílias desenvolveram "amortecedores" para lidar com os conflitos familiares e transformaram situações de crise em momentos de enfrentamento e fortalecimento (Walsh, 2005). Esta hipótese, no entanto, não pôde ser confirmada neste estudo.

Os dados de desesperança podem também indicar uma tendência das entrevistadas em responder ao questionário motivadas por uma esperança desejada e não real, já que Beck alerta para o fato de que a BHS aplicada em pacientes sem sintomatologia depressiva pode gerar uma "confusão com desejabilidade social" (p. 7).

No tocante aos transtornos psiquiátricos, $23 \% \quad(n=13) \quad$ da amostra estudada apresentou depressão e 5\%(n=3) transtorno de ansiedade, sendo que $16 \% \quad(n=9)$ dessas entrevistadas apresentaram desesperança moderada e grave. Segundo os autores, uma desesperança moderada com escore 9 ou mais na BHS deve ser complementada por uma investigação mais ampla sobre depressão e ideação suicida (Beck, 2001).

Os resultados encontrados neste estudo deixam clara a necessidade de intervenção em familiares de dependentes químicos objetivando diminuir a possibilidade de ideação suicida principalmente nos casos associados com um diagnóstico psiquiátrico.

Os achados deste trabalho não podem ser generalizados, uma vez que foi estudada uma amostra pequena, com localização geográfica restrita à região periférica de São Paulo, mas levanta uma questão importante no que se refere à percepção da qualidade de vida e ao grau de desesperança de familiares de dependentes químicos, principalmente quando esses dados estão associados com transtornos psiquiátricos.

\section{Conclusão}

Apesar do fator "convivência com um dependente químico" afetar os envolvidos nesta relação, a amostra estudada apresentou um resultado satisfatório no que se refere ao nível de desesperança, porém o grupo com desesperança moderada/grave desperta preocupações e demonstra que há a necessidade de intervenção nessa população, principalmente quando associado com a presença de transtornos psiquiátricos.

A percepção de qualidade de vida revela que as entrevistadas priorizam melhorias no âmbito das necessidades avaliadas pelo domínio meio ambiente, mais do que nos aspectos de cunho pessoal medido pelos domínios: social, físico e psicológico.

\section{Referências}

Adeodato, V. G., Carvalho, R. R. C., Siqueira, V. R. \& Souza, F. G. M., (2005). Qualidade de vida e depressão em mulheres vítimas de seus parceiros. Rev. Saúde Pública, 39(1), 108-113. 
Associação Nacional de Empresas de Pesquisa (ANEP). (1997). Critério de Classificação Econômica Brasil: Manual. São Paulo.

Beck, A. T. (2001). Manual da versão em português das Escalas de Beck (J.A. Cunha, Trans.). São Paulo: Casa do Psicólogo. (Original work published 1993).

Carr, A. (2004). Thematic review of family therapy journals in 2003. The Journal of family therapy, 430-445.

Castro M. G., Oliveira, M. S., Moraes J. F. D., Miguel A. C. \& Araujo, R. B. (2007). Qualidade de vida e gravidade da dependência de tabaco. Rev. Psiquiatria Clínica., 34(2), 61-67.

Edwards, G., Marsall, E. J. \& Cook, C. C. H. (1999). O tratamento do alcoolismo. Porto Alegre: Artes Médicas.

Figlie, N. B., Fontes, A., Moraes, E. \& Payá, R. (2004). Filhos de dependentes químicos com fatores de risco bio-psicosociais: necessitam de um olhar especial?. Rev. Psiquiatria Clínica, 31(2), 53-62.

Fleck M. P. A., Chachamovich E. \& Trentini C. M. (2003). Projeto WHOQOL-OLD: método e resultados de grupos focais no Brasil. Rev. Saúde Pública, 37(6), 793-9.

Fleck M. P. A., Louzada S., Xavier M., Chachamovich E., Vieira G., Santos L. \& Pinzon V. (2000) Aplicação da versão em português do instrumento WHOQOL-bref. Rev. Saúde Pública, 34(2), 178-83.

Fleck, M. P. A., Lima A. F. B. S., Louzada S., Schestatsky G., Henriques A. \& Borges V. X. (2002). Associação entre sintomas depressivos e funcionamento social em cuidados primários à saúde. Rev. Saúde Pública, 36(4), 431-8.

Fundação Seade; Secretaria de Estado da Saúde, (2001-2005). IBGE. Censo demográfico 1991 e 2000; Seade, 1991-2004. Nota: População em $1^{\circ}$. de Julho. - Instituto Geográfico e Cartográfico - IGC; IBGE; Seade, 1991-2004. Obtido em World Wide Web http://www.seade.gov.br.

Gorestein C. \& Andrade, H. (1998). Inventário de depressão de Beck: propriedades psicométricas da versão em português. Rev. Psiquiatria Clínica, $25,245-50$.

Henggeler, S. W., Melton, G. B. \& Smith, L. A. (1992). Family peservation using multisystemic therapy: an effective alternative to incarcerating serious juvenile offenders. $J$ Consult Clinical Psychol, 60, 953-61.

Hinkly, D. \& Laranjeira, R. (2002). Avaliação da densidade de pontos de vendas de álcool e sua relação com a violência. Rev. Saúde Pública, 36(4), 455-461.

Kernas-Bodkin, J. N. \& Leonard K. E. (2005). Alcohol involvement and marital quality in the years of marriage: a longitudinal growth curve analysis. Alcohol Clinical Experimental Research, 29(12), 2123-2134.

Levene, H. (1960). In contributions to probability and statistics: essays in honor of harold hotelling, Stanford University Press, pp. 278-292.

Lima, A. F. B. S. (2002). Qualidade de vida em pacientes do sexo masculino dependentes de álcool. Dissertação de mestrado. Porto Alegre: Universidade Federal do Rio Grande do Sul.

Minkoff, K., Bergman, E., Beck, A. T. \& Beck, R. (1973). Hopelessness, depression and attempted suicide. American Journal of Psychiatry, 130(4), 455-459.

Orley J., Saxena S. \& Herrman H. (1998). Quality of Life mental illness: reflections from the perspective of the WHOQOL. British Journal of Psychiatry, 172, 291-3.

Schenker, M. \& Minayo, M. C. S. (2004). A importância da família no tratamento do uso abusivo de drogas: uma revisão da literatura. Cad. Saúde Pública, 20(3).

Tempier R., Boyer R., Lambert J., Mosier K. \& Duncan C. R. (2006). Psychological distress among females spouses of male at risk drinkers. Alcohol, 40(1), 41-49.

The WHOQOL Group (1998). Development of the World Health Organization. Whoqol bref. Quality of life assessment psycological medicine, 28, 551-558.

Tobo N. I. V. \& Zago M. M. F. (2005). El sufrimento de la esposa en la convivência con el consumidor de bebidas alcohólicas. Rev. Latino-am Enfermagem, 13, 806-12.

Walsh, F. (2005). Fortalecendo a Resiliência Familiar. São Paulo: Roca. 
Recebido em maio de 2008 Reformulado em outubro de 2008 Aprovado em janeiro de 2009

Sobre os autores:

Antonio Teulberto Mesquita Aragão é graduado em Psicologia pela Universidade Metodista de São Paulo (1988) com extensão em Adm. de Recursos Humanos pela Fund. Getúlio Vargas (1995), especialização em Promoção da Saúde na Prevenção de Dependência de Álcool e Drogas pela Universidade Federal de São Paulo - UNIAD (2007).

Elizabete Milagres é assistente social e especialista em Dependência Química pela UNIAD-UNIFESP.

Neliana Buzi Figlie é psicóloga, especialista em Dependência Química pela UNIAD-UNIFESP, mestre em Saúde Mental, doutora em Ciências pelo depto. de Psiquiatria da Universidade Federal de São Paulo e professora orientadora do Curso de Pós-Graduação em Psiquiatria da UNIFESP. 